\title{
Review
}

\section{Tipping Points and Endogenous Determinants of Nigrostriatal Degeneration by} MPTP

\author{
Stefan Schildknecht, ${ }^{1, \star}$ Donato A. Di Monte, ${ }^{2}$ Regina Pape, ${ }^{1}$ \\ Kim Tieu, ${ }^{3}$ and Marcel Leist ${ }^{1}$
}

The neurotoxicant 1-methyl-4-phenyl-1,2,3,6-tetrahydropyridine (MPTP) causes a Parkinson's disease (PD)-like syndrome by inducing degeneration of nigrostriatal dopaminergic neurons. Studies of the MPTP model have revealed the pathomechanisms underlying dopaminergic neurodegeneration and facilitated the development of drug treatments for PD. In this review, we provide an update on MPTP bioactivation and biodistribution, reconcile the distinct views on energetic failure versus reactive oxygen species (ROS) formation as main drivers of MPTP-induced neurodegeneration, and describe recently identified intrinsic features of the nigrostriatal system that make it particularly vulnerable to MPTP. We discuss these new perspectives on the endogenous tipping points of tissue homeostasis and the drivers responsible for vicious cycles in relation to their relevance for the development of novel intervention strategies for PD.

\section{MPTP: An Experimental Parkinsonian Toxicant}

Almost four decades ago, the neurotoxicant MPTP was identified as an illicit drug contaminant that can cause symptoms and signs in humans similar to those observed in idiopathic PD [1]. Studies during the 1980s elucidated the basic mechanisms of toxicity of MPTP, which has since become the most-studied experimental neurotoxicant. It is now textbook knowledge that the protoxicant MPTP is metabolized by astrocytic monoamine oxidase-B (MAO-B; see Glossary) to generate the active metabolite 1-methyl-4-phenylpyridinium $\left(\mathrm{MPP}^{+}\right)$(Figure 1). $\mathrm{MPP}^{+}$is taken up into neurons by dopamine (DA) transporters (DATs) [2-5]. Cytosolic MPP ${ }^{+}$ is accumulated in catecholaminergic storage vesicles by vesicular monoamine transporter2 (VMAT-2) and in mitochondria through membrane potential-dependent uptake [6]. MPP ${ }^{+}$ accumulates in the mitochondrial matrix and inhibits complex I of the respiratory chain, leading to an impairment in mitochondrial ATP generation and an increase in superoxide $\left(\bullet \mathrm{O}_{2-}\right)$ formation [6-8]. Furthermore, $\mathrm{MPP}^{+}$uptake into DA neurons triggers vesicular DA release, which results in DA autoxidation and free radical formation (Figure 2). In the presence of iron and $\mathrm{H}_{2} \mathrm{O}_{2}$, extracellular DA can be oxidized to form 6-hydroxy-DA. This toxicant can undergo cyclization to yield aminochrome, which is capable of directly inhibiting complex I [9]. Thus, oxidative stress and energy failure are involved intricately in a vicious cycle. ATP depletion triggered by MPP ${ }^{+}$emerged as insufficient to trigger cell death [10]. A growing body of evidence indicates a more significant role of ROS generation as consequence of complex I inhibition than initially anticipated $[11,12]$. For instance, mice overexpressing copper/zinc superoxide

\section{Trends}

PD-associated motor deficits are characterized by nigrostriatal dopaminergic-neuron loss.

The parkinsonian protoxicant MPTP is activated in the central nervous system (CNS) by astrocytic monoamine oxidase-B to form the active toxicant 1-methyl-4-phenylpyridinium (MPP ${ }^{+}$).

Membrane-impermeable $\mathrm{MPP}^{+}$is taken up by neurotransmitter transporters into catecholaminergic neurons.

Inside cells, $\mathrm{MPP}^{+}$inhibits mitochondrial complex I, reducing mitochondria ATP output and favoring ROS formation.

In MPTP-exposed brains, different catecholaminergic populations show large sensitivity differences, with a distinct nigrostriatal dopaminergicneuron degeneration, reflecting the degeneration pattern in PD.

Intrinsic factors, such as MPP ${ }^{+}$uptake and/or vesicular-sequestration kinetics, neuronal morphology, and intracellular $\mathrm{Ca}^{2+}$ handling, distinguish sensitive from resistant neuronal subpopulations in the MPTP model and in PD.

${ }^{1}$ In vitro Toxicology and Biomedicine, Department of Biology, University of Konstanz, D-78457 Konstanz,

Germany

${ }^{2}$ German Center for

Neurodegenerative Diseases (DZNE), Sigmund-Freud-Strasse 27, D-53127 Bonn, Germany 
<smiles>CN1CC=C(c2ccccc2)CCN1</smiles><smiles>[CH]1CC1</smiles>

${ }^{3}$ Department of Environmental \& Occupational Health, Florida International University, Miami, FL 33199, USA

${ }^{*}$ Correspondence:

Stefan.Schildknecht@uni-konstanz.de (S. Schildknecht).

Figure 1. Structures of 1-Methyl-4-Phenyl-1,2,3,6-Tetrahydropyridine (MPTP) and Its Metabolites. The intermediate 1-methyl-4-phenyl-2,3-dihydropyridinium $\left(\mathrm{MPDP}^{+}\right)$is generated through a two-electron oxidation, catalyzed by monoamine oxidase (MAO). Under physiological conditions, $\mathrm{MPDP}^{+}$partially exists in its conjugate base form, 1,2MPDP. Given that it is lipophilic and uncharged, 1,2-MPDP can readily cross membranes without the need for a transporter. In a second, nonenzymatic step, the intermediate undergoes autoxidation to form the stable active toxicant 1-methyl-4-phenylpyridinium $\left(\mathrm{MPP}^{+}\right)$.

dismutase (Cu/Zn-SOD) displayed higher resistance against MPTP [13], while knockdown of endogenous Cu/Zn-SOD elevated the sensitivity of nigrostriatal DA neurons towards MPTP [14] (Figure 3).

Recent groundbreaking studies on MPTP models have provided data that cannot be satisfactorily explained by standard textbook knowledge regarding MPTP toxicokinetics and mode of action. Take, for example, modulation of neuroinflammation-induced damage: blockade of IL$1 \beta$, TNF- $\alpha$, or IFN- $\gamma$ signaling prevented MPTP neurotoxicity, despite ongoing MPP ${ }^{+}$inhibition of mitochondrial complex I [15-17]. Another example is related to mitochondrial fission and/or fusion. Genetic silencing or pharmacological suppression of dynamin-like protein-1 (DLP-1), a mitochondrial fission-associated molecule, prevented mitochondrial dysfunction and neurodegeneration, despite $\mathrm{MPP}^{+}$-dependent inhibition of mitochondrial complex I [18-21]. Moreover, adenosine $A_{2 A}$ receptor antagonists were reported to protect against MPTP-triggered neurodegeneration, but the underlying mechanisms remain inadequately characterized [22]. Another set of questions arises from the cell type specificity of MPTP and/or MPP ${ }^{+}$-induced damage: motor deficits in PD and in MPTP models correlate closely with nigrostriatal DA- 


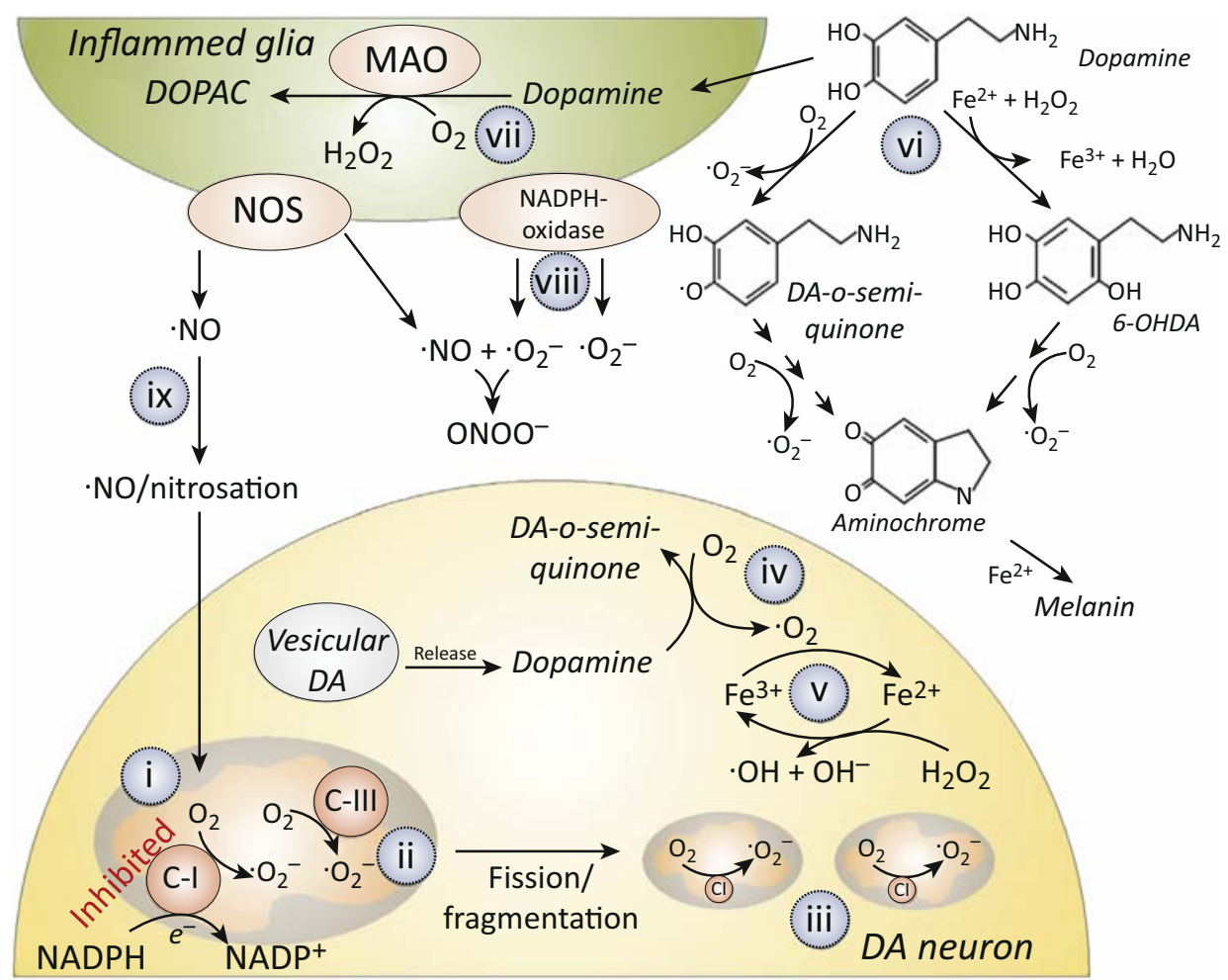

Trends in Pharmacological Sciences

Figure 2. Free Radical Sources. 1-Methyl-4-phenylpyridinium $\left(\mathrm{MPP}^{+}\right)$primarily acts as an inhibitor of mitochondria complex I (C-I). At this site, $\mathrm{MPP}^{+}$exposure stimulates ${ }^{\circ} \mathrm{O}_{2}$ - generation (i). Elevated free radical levels can lead to oxidative modifications of $\mathrm{C}$-I and of mitochondrial complex III (C-III) (ii). This increases and perpetuates $\bullet \mathrm{O}_{2}$ - formation by the respiratory chain. Consequently, mitochondrial fragmentation is accelerated, leading to increased $\bullet \mathrm{O}_{2-}$ formation by fragmented mitochondria (iii). $\mathrm{MPP}^{+}$also triggers vesicular dopamine (DA) release into both the cytosol and the synaptic cleft. Cytosolic DA undergoes autoxidation, which increases $\bullet \mathrm{O}_{2}$ - formation (iv). The $\bullet \mathrm{O}_{2}$ - radical can be dismutated to form $\mathrm{H}_{2} \mathrm{O}_{2}$, which, in the presence of iron, can initiate the Fenton reaction and drive the Haber-Weiss cycle to form highly reactive hydroxyl radicals $(\bullet \mathrm{OH})(\mathrm{v})$. Extracellular DA can also undergo autoxidation and thereby favor $\bullet \mathrm{O}_{2-}$ formation. In the presence of iron and $\mathrm{H}_{2} \mathrm{O}_{2}$, extracellular DA is oxidized to form the toxicant 6-hydroxy-DA (6-OHDA), whose subsequent cyclization generates aminochrome and $\bullet \mathrm{O}_{2-}$ (vi). Aminochrome can directly inhibit $\mathrm{C}-\mathrm{I}$ and, thus, leads to a selfperpetuating cycle. Extracellular DA, accumulated by astrocytes, can be detoxified by glial monoamine oxidase (MAO), a reaction that involves $\mathrm{H}_{2} \mathrm{O}_{2}$ generation (vii). Glial cells, activated by inflammatory stimuli or degenerating neurons, can serve as a potent source of $\bullet \mathrm{O}_{2-}$, formed by, for example, NADPH oxidases (viii). Glial cells and neurons are also sources of nitric oxide $(\bullet N O)$, generated by either constitutively expressed NO synthase (NOS)-1 or inducible NOS-2 (ix). A fitting example of reactive oxygen species (ROS) function is the finding that mice were protected against 1-methyl-4phenyl-1,2,3,6-tetrahydropyridine (MPTP) toxicity after overexpression of the antioxidative enzyme Cu/Zn-superoxide dismutase (Cu/Zn-SOD); conversely, endogenous Cu/Zn-SOD knockdown elevated DA neuron MPTP sensitivity. However, the relative contribution of different ROS sources warrants further consideration when interpreting the results obtained using diverse MPTP/MPP ${ }^{+}$models and extrapolating the results to PD. Mitochondrial ROS generation is a more complex cell biological process than previously assumed: MPTP and $\mathrm{MPP}^{+}$lead to mitochondrial fission through oxidant effects on various proteins, such as dynamin-like protein-1 (DLP-1), and mitochondrial fragmentation increases ROS output. Notably, the tipping points of all processes linked to oxidative stress and disturbed cellular proteostasis are dependent on counter-regulations (antioxidants, chaperones, etc.). These differ strongly between experimental systems (even from mouse strain to mouse strain), which helps to explain why the literature is often apparently contradictory, with certain findings being challenging to reproduce, and why predictions from models to the human disease are associated with large uncertainty.

neuron degeneration and the concomitant decline in striatal DA levels [23]. This has obscured the finding that MPTP also markedly affects DA neurons of the mesocortical system or hypothalamic tuberomammillary nucleus [24]. Furthermore, DAT-mediated toxicant uptake does not explain why MPTP spares, for example, DA neurons of the ventral tegmental area

\section{Glossary}

Cytochrome P450 oxidase (CYP2D6): involved in the metabolism and elimination of xenobiotics.

Dopamine transporter (DAT): expressed by dopamine neurons; enables re-uptake of synaptic dopamine to terminate its signaling function. DAT also allows uptake of $\mathrm{MPP}^{+}$, thus contributing to its preferential toxicity in these neurons. Monoamine oxidase (MAO): catalyzes the oxidative deamination and inactivation of monoaminergic neurotransmitters. MAO also catalyzes the activation of MPTP to form the toxicant MPP ${ }^{+}$

Organic cation transporter (OCT): allows passive transport of organic cations (incl. MPP ${ }^{+}$) across membranes.

Vesicular monoamine transporter 2 (VMAT-2): transport of neurotransmitters and $\mathrm{MPP}^{+}$from the cytosol into synaptic vesicles. 


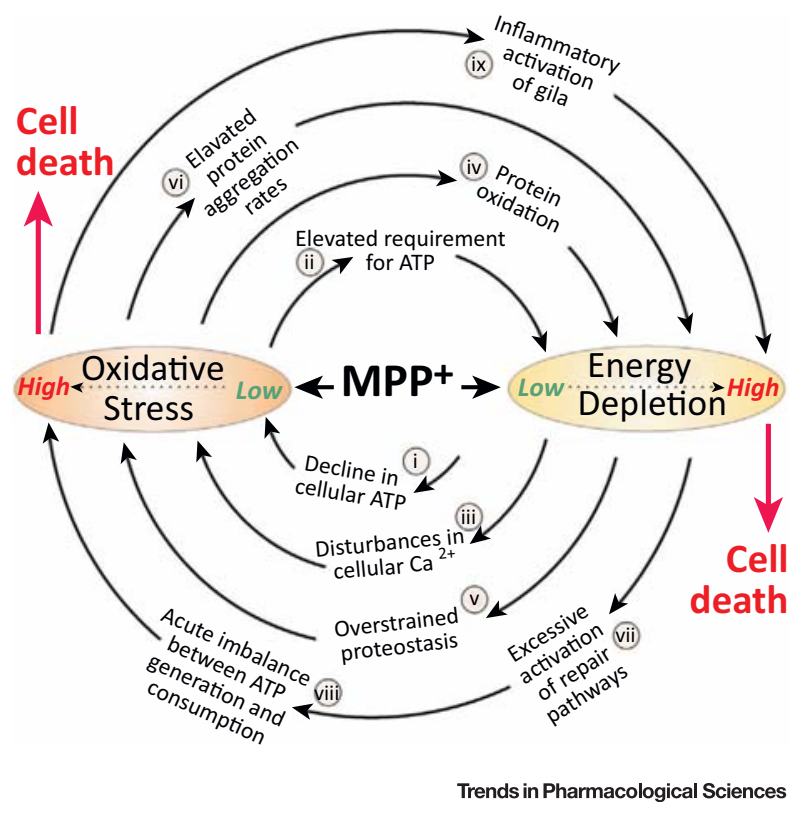

Figure 3. 1-Methyl-4-Phenylpyridinium $\left(\mathrm{MPP}^{+}\right)$As a Trigger of Vicious Cycles. Binding of $\mathrm{MPP}^{+}$to complex inhibits mitochondrial ATP synthesis and triggers $\bullet \mathrm{O}_{2-}$ formation (i). These events initiate a series of self-amplifying and selfperpetuating processes that lead to the demise of the cell through a vicious cycle. To compensate for elevated $\bullet \mathrm{O}_{2}$ formation, the cell must maintain antioxidant systems, which further burdens the energy budget (ii). As a result of $\mathrm{Ca}_{v} 1.3-$ mediated pacemaking, nigrostriatal dopamine (DA) neurons face a constant influx of extracellular $\mathrm{Ca}^{2+}$. Inappropriate control of cellular $\mathrm{Ca}^{2+}$ pools, as a consequence of an already stressed ATP budget (iii), can lead to $\mathrm{Ca}^{2+}$ accumulation in mitochondria and thereby to an increase in mitochondrial $\bullet \mathrm{O}_{2}$ - generation. Such elevated rates of ROS formation lead to increased rates of oxidative modifications of proteins, lipids, and DNA (iv), which further reduce key cellular functions, such as mitochondrial ATP synthesis. Moreover, cellular functions such as proteostasis are reduced as a consequence of inappropriate ATP generation (v). This leads to an accumulation of oxidatively modified and misfolded proteins (vi), which further compromises mitochondrial function. Activation of cellular repair pathways leads to an additional demand for ATP (vii), further enhancing the already existing imbalance between ATP consumption and generation (viii). All of the aforementioned events ultimately result in the initiation of cell damage or death, and this leads to an inflammatory activation of surrounding glial cells. Glial-derived reactive oxygen and nitrogen species further increase oxidative stress in the remaining neurons (ix).

(VTA). MPP ${ }^{+}$uptake by other catecholamine transporters explains the pronounced degeneration of noradrenergic neurons of the locus coeruleus in the MPTP model [25], but is inconsistent with the resistance of serotonergic, MAO-B-containing neurons [26].

These examples of outstanding questions and inconsistencies illustrate how the simplified textbook view is insufficient for explaining the observations made using MPTP models (see Outstanding Questions). Conversely, new insights into MPTP metabolism and biodisposition, together with expanding knowledge regarding the intrinsic features of neuronal subpopulations primarily affected in the MPTP model and PD, have led to a more precise understanding of the underlying mechanisms. Here, we review these novel findings to facilitate the interpretation of data obtained using MPTP and/or MPP ${ }^{+}$models, and address the critical question of how observations made in these test systems can broaden our understanding of pathogenetic processes in PD.

\section{Why Is MPP ${ }^{+}$Not Accumulating within Astrocytes?}

In the central nervous system (CNS), MPTP is converted to $\mathrm{MPP}^{+}$almost entirely by MAO-B, a mitochondrial outer-membrane enzyme expressed predominantly in astrocytes and, to a minor extent, in serotonergic neurons $[27,28]$. Being charged, $\mathrm{MPP}^{+}$cannot diffuse across cell 
membranes, and would be expected to accumulate and cause toxicity within MPP ${ }^{+}$-producing cells. This contrasts with the lack of astroglial damage typically observed upon MPTP exposure $[29,30]$, although astrocytes are not $\mathrm{MPP}^{+}$resistant. In astrocytes, genetically modified to express DAT, MPP ${ }^{+}$accumulates intracellularly and elicits a toxic response comparable to that in DA neurons $[31,32]$. These findings suggest that $\mathrm{MPP}^{+}$toxicity in any cell type is determined mainly by the toxicant concentration reached inside mitochondria. Cells have been widely demonstrated to exhibit distinct susceptibilities largely due to variations in $\mathrm{MPP}^{+}$uptake across the plasma membrane, availability of export mechanisms, and intracellular deposition in organelles. Astrocytes might be spared from MPTP-induced cell death because of an efficient export of MPTP metabolites [32] (Figure 4).

One key carrier that enables $\mathrm{MPP}^{+}$equilibration across cellular membranes is the organic cation transporter-3 (OCT-3) [33]. OCT-3 is preferentially expressed in glial cells located near the DA neurons, and OCT-3-deficient mice were highly resistant to MPTP toxicity [33]. This suggests a critical role of OCT-3 in MPTP-dependent neurodegeneration, although the mechanistic basis is complex because OCT-3 allows not only the export of cationic compounds, such as $\mathrm{MPP}^{+}$or monovalent paraquat, but also their uptake [33,34]. Depending on the brain region and phase of MPTP metabolism, OCT-3-expressing cells might act as a transient 'sink' for high levels of extracellular MPP ${ }^{+}$, or OCT-3 might allow $\mathrm{MPP}^{+}$efflux from cells in which MPP is highly accumulated. This complex $\mathrm{MPP}^{+}$distribution is also affected by the relative affinities of the transporters, as exemplified by the $\mathrm{K}_{m}$ of DAT being lower than that of OCT-3 (Figure 4). Near DAT-expressing neurons, this lower $\mathrm{K}_{\mathrm{m}}$ of DAT for $\mathrm{MPP}^{+}$favors $\mathrm{MPP}^{+}$uptake and retention within dopaminergic neurons [33].

MPTP metabolites are also exported through a transporter-independent mechanism [32]. Understanding how $\mathrm{MPP}^{+}$is removed from astrocytes lacking OCT or related transporters requires comprehension of the metabolic steps involved in MPTP bioactivation. This twostep process starts with MAO-B-catalyzed two-electron $\alpha$-carbon oxidation of MPTP, which generates the unstable intermediate 1-methyl-4-phenyl-2,3-dihydropyridinium $\left(\mathrm{MPDP}^{+}\right)$ $[35,36]$. In the second step, which is nonenzymatic, MPDP ${ }^{+}$undergoes autoxidation to form the stable $\mathrm{MPP}^{+}\left[37\right.$ ] (Figures 1 and 4). Although a role of $\mathrm{MPDP}^{+}$in extracellular MPP ${ }^{+}$ formation and accumulation was suggested in early MPTP studies [3,37], MPDP ${ }^{+}$was only recently measured in brain tissue and identified as a transporter-independent export metabolite in its uncharged, membrane-permeable free-base form: 1,2-MPDP [32]. Specific conditions in brain tissues have been shown to stabilize the intermediate intracellularly and concurrently promote its conversion to $\mathrm{MPP}^{+}$extracellularly. The intracellular milieu is characterized by lower oxygen tension due to mitochondrial consumption and moderately more acidic conditions compared with the extracellular environment [38]. Whereas these conditions prevent $\mathrm{MPP}^{+}$formation when $\mathrm{MPDP}^{+} / 1,2-\mathrm{MPDP}$ is inside cells, conversion into $\mathrm{MPP}^{+}$is promoted by the more alkaline and oxygen-rich environment and the iron-containing complexes in the extracellular space [32] (Figure 4). Identification of $\mathrm{MPDP}^{+} / 1,2-\mathrm{MPDP}$ as a membrane-permeable MPTP metabolite and elucidation of the extracellular conditions that favor its autoxidation to $\mathrm{MPP}^{+}$provide a convincing mechanism for explaining the resistance of MPTP-converting astrocytes (Figure 4). The rapid clearance of extracellular MPP ${ }^{+}$out of the brain guarantees a constant steep concentration gradient between intracellular and extracellular compartments and, thus, a sustained efflux of $\mathrm{MPDP}^{+} / 1,2-\mathrm{MPDP}$ from astrocytes. This molecular explanation for preferential $\mathrm{MPP}^{+}$formation in the extracellular space is also required for understanding the mechanism underlying the long-recognized DAT-dependent uptake of extracellular $\mathrm{MPP}^{+}$into nigrostriatal DA neurons [32]. The notion that a large fraction of $\mathrm{MPP}^{+}$formed in the brain prevails in the extracellular space is support by microdialysis and mass spectrometry studies in MPTP-exposed mice and rats $[39,40]$ : $\mathrm{MPP}^{+}$levels rose rapidly throughout the brain, but MPP ${ }^{+}$was also cleared rapidly $(1-2 \mathrm{~h})$ 


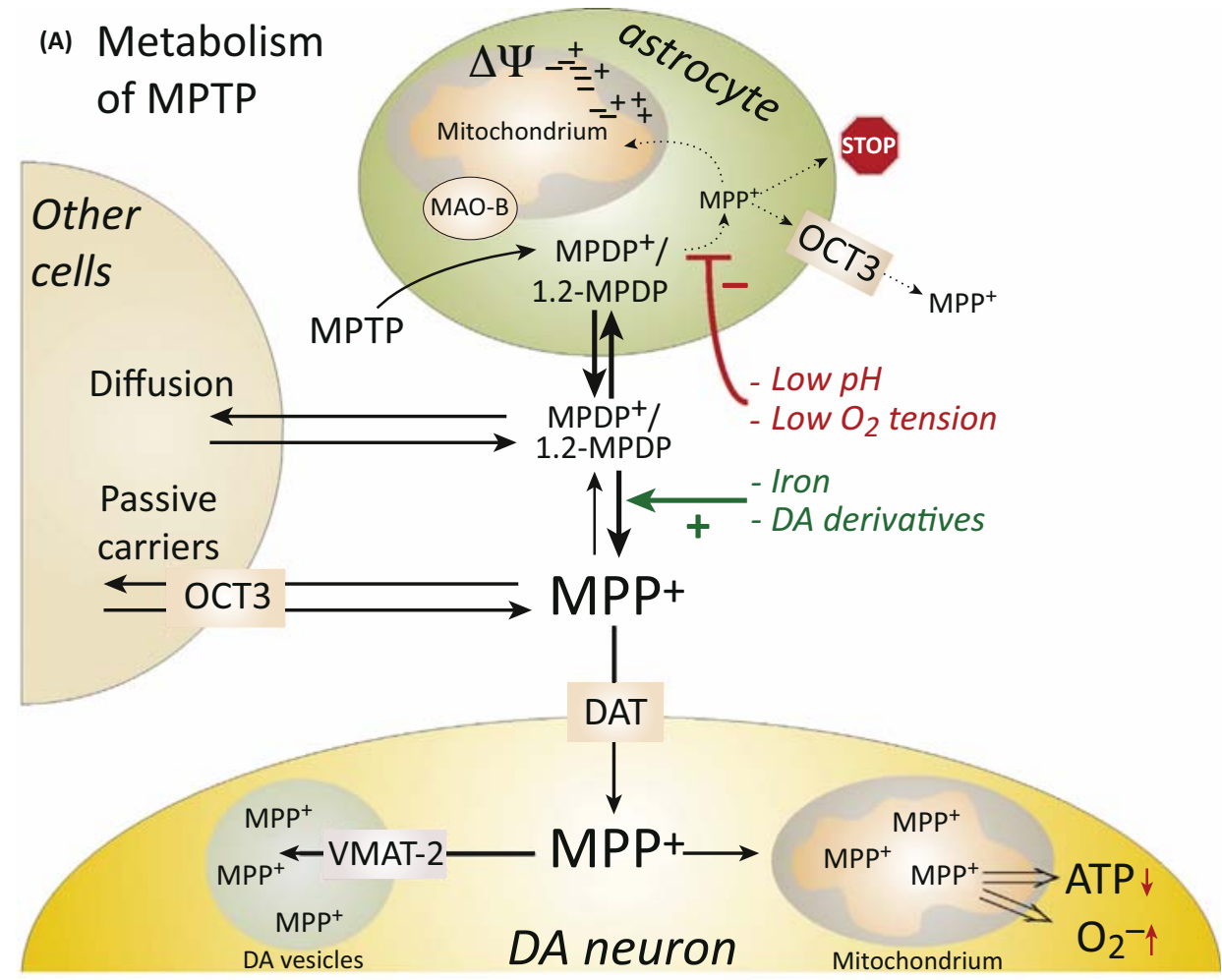

(B) Facilitated diffusion

$\Delta \Psi$ Mitochondrial

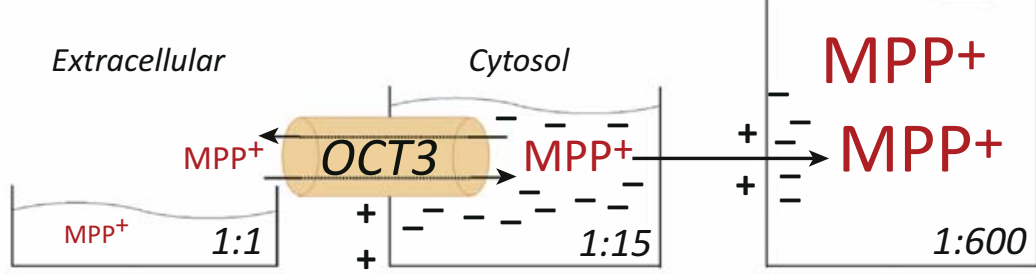

(c) Transporter-mediated uptake

$\mathrm{MPP}^{+} \quad 1: 1$

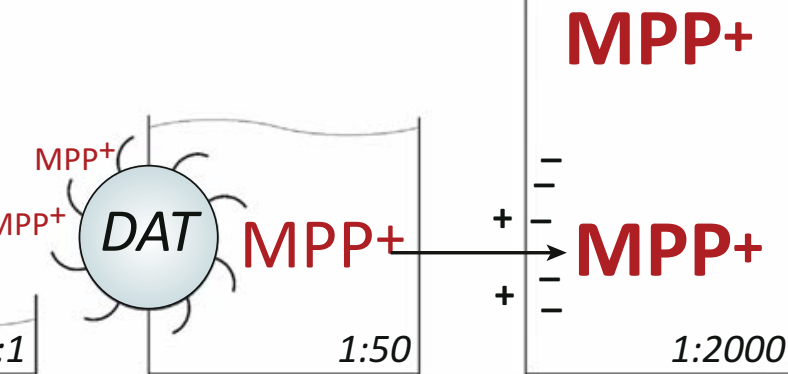

Figure 4. Metabolism of 1-Methyl-4-Phenyl-1,2,3,6-Tetrahydropyridine (MPTP) and Distribution of 1Methyl-4-Phenylpyridinium $\left(\mathrm{MPP}^{+}\right)$. (A) In the brain, most MPTP is converted by glial monoamine oxidase-B (MAO-B) into the intermediate MPDP +1 ,2-MPDP, which freely diffuses across biological membranes. Once outside astrocytes, the intermediate might either diffuse into other cells or undergo nonenzymatic autoxidation to form the stable toxicant $\mathrm{MPP}^{+}$. Several factors favor a preferential formation of $\mathrm{MPP}^{+}$in the extracellular space: inside cells, the relatively 
in most areas, except regions rich in neurons that actively accumulate $\mathrm{MPP}^{+}$via their catecholamine transporters.

Which Enzymes in Neurons or Astrocytes Contribute to MPTP Oxidation? Chiba et al. [35] discovered the MAO-dependent activation of MPTP to form MPP ${ }^{+}$. Knockout studies and pharmacological intervention studies using MAO-A-/MAO-B-selective inhibitors indicated that MAO-B has a dominant role in MPTP activation in the CNS [41]. An alternative activation pathway, MPTP conversion by cytochrome P-450 2D6 (CYP2D6), was also suggested $[42,43]$. Indeed, genetic association studies revealed a link between a polymorphism at the locus CYP2D6 and PD susceptibility [44]. However, biochemical activity studies indicated that high CYP2D6 activities were correlated with a diminished risk of PD $[45,46]$. A potential molecular explanation for these observations is the detoxification of endogenously formed $\beta$-carbolines and isoquinolines and inactivation of pesticides by CYP2D6 $[42,47]$.

The aforementioned observations in PD raise this question: does CYP2D6 have a protective or toxicity-enhancing role in the MPTP model? The major metabolites produced by CYP2D6, 1methyl-4-(4-hydroxyphenyl)-1,2,3,6-tetrahydropyridine (OH-MPTP; $p$-hydroxylation) and 4phenyl-1,2,3,6-tetrahydropyridine (PTP; $N$-demethylation) [47], are not neurotoxic, and only approximately $10 \%$ of MPTP is transformed into $\mathrm{MPDP}^{+}$[48]. This agrees with the finding that the cytochrome P450 inhibitor diethyldithiocarbamate increases MPTP toxicity in mice [49]. Furthermore, CYP2D6 upregulation protected against MPTP also in vitro [50]. Analysis of the respective contributions of MAO-B and CYP2D6 to MPTP oxidation revealed that the oxidation rate by MAO is approximately threefold higher [48]. The fact that CYP2D6 has a minor role only in $\mathrm{MPP}^{+}$formation is supported by results obtained using MAO-B-knockout mice, which were almost completely protected against MPTP [41]. Thus, even if CYP2D6 contributes to MPDP ${ }^{+}$ formation, the extent of this reaction would likely be insufficient to damage and kill DA neurons. In conclusion, CYP2D6 expression in the nigrostriatal system can be regarded as a protective factor in idiopathic PD and in the MPTP model.

\section{How Does Sequestration inside Neurons Determine MPP ${ }^{+}$Toxicity?}

Efficient inhibition of isolated complex I of the mitochondrial respiratory chain was found to require 10-20 $\mathrm{mM} \mathrm{MPP}^{+}$[6], concentrations that are 500-2000-fold higher than those measured in brain homogenates after MPTP treatment $[51,52]$. This raises the question of how MPP ${ }^{+}$ is accumulated intracellularly at concentrations sufficient for complex I inhibition (Figure 4).

lower $\mathrm{pH}$ and low oxygen tension contribute to a stabilization of the intermediate, whereas in the extracellular space, the availability of either catalytically active iron-containing complexes or dopamine (DA) autoxidation products can strongly accelerate the autoxidation of $\mathrm{MPDP}^{+}$into $\mathrm{MPP}^{+}$. Extracellular availability of $\mathrm{MPP}^{+}$is a prerequisite for its cell specific, transporter-dependent accumulation in catecholaminergic neurons. In DA neurons, cytosolic $\mathrm{MPP}^{+}$is either actively accumulated in neurotransmitter vesicles by vesicular monoamine transporter-2 (VMAT-2), or is enriched in the mitochondrial matrix, driven by the mitochondrial transmembrane potential. In mitochondria, MPP ${ }^{+}$inhibits complex I of the respiratory chain, which leads to the limitation of mitochondrial ATP production and to an increase in $\bullet \mathrm{O}_{2-}$ formation at complex I. MPP ${ }^{+}$distribution in the brain is also affected by passive transporters, such as organic cation transporter- 3 (OCT-3). Such bidirectional transporters allow a membrane potential-dependent uptake of MPP ${ }^{+}$. Under conditions of low extracellular $\mathrm{MPP}^{+}$, these transporters also facilitate the export of intracellular $\mathrm{MPP}^{+}$. (B) Transport of extracellular MPP ${ }^{+}$ into the mitochondrial matrix requires passage across the (1) cell membrane and the (2) inner mitochondrial membrane. Passive transporters allow an intracellular accumulation of the cation $\mathrm{MPP}^{+}$, according to the Nernst equation. At a given membrane potential of $-70 \mathrm{mV}$, an approximately 15 -fold concentration of $\mathrm{MPP}^{+}$can theoretically be achieved. (C) Active transporters, such as the DA transporter (DAT), enable a unidirectional build-up of MPP ${ }^{+}$in the cytosol against a concentration gradient by a factor of approximately 1:50. In a second step, cytosolic MPP ${ }^{+}$is accumulated in the mitochondrial matrix, mostly driven by the transmembrane potential of the inner mitochondrial membrane. This step permits an additional concentration by a factor of approximately $1: 40$. When extracellular $\mathrm{MPP}^{+}$is present in the low micromolar range, OCT expression is inadequate to enable quantitative complex I inhibition, whereas at the same concentrations, DAT expression allows millimolar toxicant concentrations to be reached in mitochondria, which is sufficient for inhibiting the respiratory chain. 
When passive transporters are present, the plasma membrane potential of $-70 \mathrm{mV}$ would lead to an approximately 15 -fold concentration of the monovalent cation $\mathrm{MPP}^{+}$intracellularly, according to the Nernst equation [32]. Moreover, isolated respiring mitochondria accumulate $\mathrm{MPP}^{+}$by a factor of approximately 40 [6]. Given that tissue $\mathrm{MPP}^{+}$concentrations of $>10 \mu \mathrm{M}$ can be reached in standard MPTP models in vivo [51,52], this two-step electrochemical accumulation would allow $\mathrm{MPP}^{+}$to reach millimolar concentrations in the mitochondrial matrix (Figure 4). However, such concentrations would be maintained only transiently: MPTP intoxication is characterized by rapid $\mathrm{MPP}^{+}$clearance from the brain (half-life in the 1-h range). Moreover, cytosolic $\mathrm{MPP}^{+}$efflux through a bidirectional carrier would reduce its mitochondrial concentration, which would lead to the sparing of cells from $\mathrm{MPP}^{+}$toxicity.

The aforementioned scenario is contrasted by what occurs in catecholamine neurons, which express two active transporters: a plasma membrane catecholamine transporter, such as DAT; and the vesicular transporter VMAT-2. Unlike the OCT-transporter-mediated bidirectional passive flux, catecholaminergic transporters drive active, unidirectional $\mathrm{MPP}^{+}$accumulation (Figure 4). Once $\mathrm{MPP}^{+}$is in the cytosol, its further sequestration, either by mitochondrial accumulation or by VMAT-2-mediated vesicular accumulation, largely determines MPP ${ }^{+}$toxicity. Whereas DAT overexpression enhanced neuronal MPTP sensitivity [53], pharmacological inhibition of DAT protected against MPTP toxicity [5]. VMAT-2 mediates vesicular MPP ${ }^{+}$ sequestration, which prevents MPP $^{+}$toxicity and, accordingly, VMAT-2 overexpression resulted in diminished toxicity [54], whereas its genetic ablation or pharmacological inhibition elevated the MPTP sensitivity of DA neurons [55]. Analysis of DAT and VMAT-2 expression in distinct neuronal populations revealed that the DAT:VMAT-2 ratio was higher in MPTP-sensitive striatal terminals of nigral DA neurons than in comparatively less sensitive VTA neurons [56]. Disturbances in vesicular storage affect not only DA neurons, but also noradrenergic neurons of the locus coeruleus. In a low-VMAT-2-expression mouse model, locus coeruleus noradrenergic neurons exhibited progressive degeneration even before the onset of nigrostriatal DA neurodegeneration [57]. Conversely, polymorphisms identified in the human VMAT-2 promoter region indicated that elevated VMAT-2 expression correlated with reduced PD risk [58,59]. Notably, mice exposed during development to dieldrin, an environmental toxicant, displayed an increased DAT:VMAT-2 ratio in the adult nigrostriatal system and, consequently, were more vulnerable to MPTP toxicity than were unexposed control mice [60]. In conclusion, not only the activity of cell surface catecholamine transporters, but also intracellular vesicular sequestration determines the widely recognized variations in MPTP sensitivity of distinct catecholaminergic populations of the brain. These factors must be carefully considered when interpreting the region-selective differences in neuronal degeneration observed in MPTP models.

\section{Features of Nigrostriatal DA Neurons That Affect the Tipping Point of MPP ${ }^{+}$ Toxicity}

As described above, mechanisms of $\mathrm{MPP}^{+}$production, accumulation, and sequestration are not the only determinants of the MPTP susceptibility of neuronal subpopulations. This concept is exemplified by the evidence that not all catecholaminergic neurons (i.e., not all neurons that can take up $\mathrm{MPP}^{+}$) are equally targeted by MPTP neurotoxicity $[23,24,26]$. Specific neuronal features have recently been identified as modulators of the selective effects of MPTP, and these susceptibility factors could also have a critical role in PD pathogenesis.

Intracellular $\mathrm{Ca}^{2+}$ and Its Influence on Energy Expenditure

Among the endogenous factors that contribute to selective neuronal degeneration in the MPTP model, dysregulation of intracellular $\mathrm{Ca}^{2+}$ homeostasis has a central role [61] (Figure 5), a conclusion supported by evidence from studies illustrating a neuroprotective influence of intracellular $\mathrm{Ca}^{2+}$ regulation [62]. Accordingly, the endogenous $\mathrm{Ca}^{2+}$-binding protein calbindin appears to promote neuroprotection: MPTP-resistant VTA neurons express higher levels of 


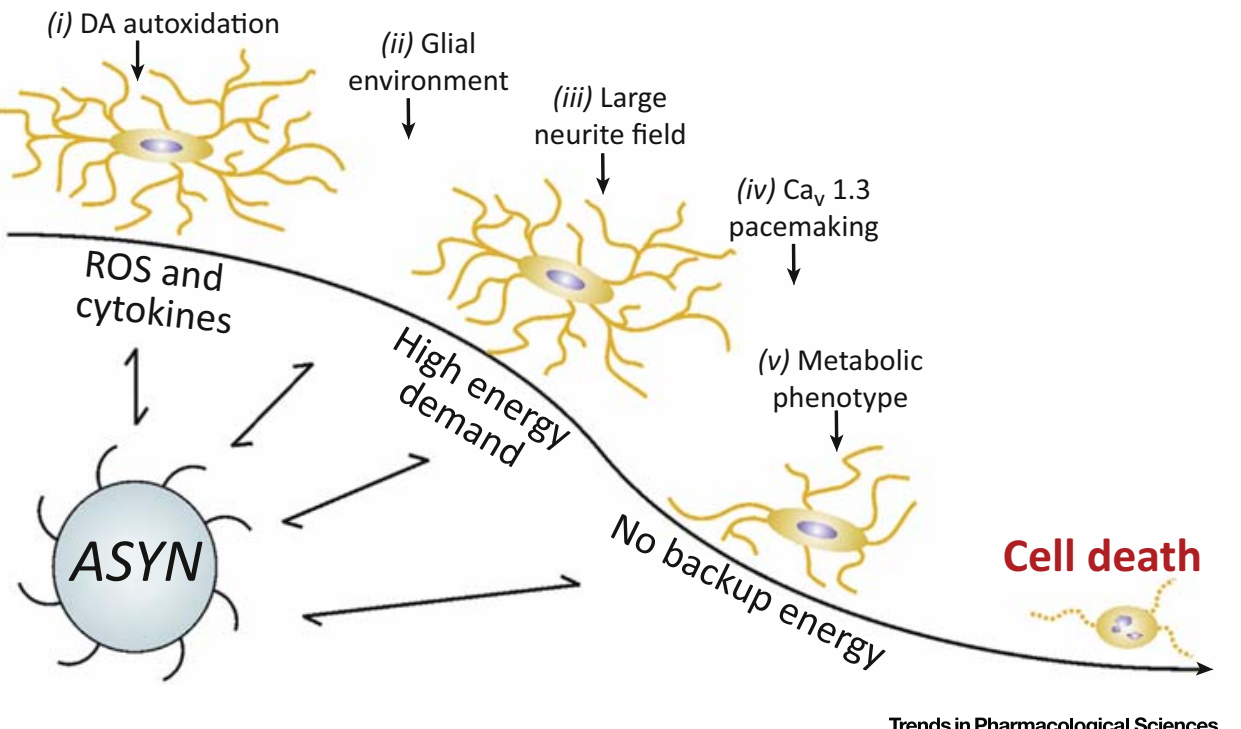

Figure 5. Endogenous Susceptibility Factors of Nigrostriatal Dopamine (DA) Neurons. Nigrostriatal DA neurons are continuously exposed to a series of stressors, such as elevated levels of free radicals, derived from DA autoxidation, (i) and from reactive oxygen and nitrogen species, which can be generated upon inflammatory activation of microglia in response to initial neuronal damage (ii). Nigrostriatal DA neurons differ from several other neuronal populations by virtue of their large total neurite length, a higher degree of neurite branching, and, consequently, a large number of synapses per neuron (iii). This leads to a particularly high energy demand, which is further increased because nigrostriatal DA neurons are autonomous pacemakers and use $\mathrm{Ca}_{v} 1.3 \mathrm{~L}$-type $\mathrm{Ca}^{2+}$ channels for pacemaking (iv). Maintenance of intracellular $\mathrm{Ca}^{2+}$ homeostasis represents a continuous challenge to the cellular ATP budget. The individual metabolic phenotype (i.e., the high reliance on mitochondrial metabolism instead of glycolysis) further compromises DA neuron survival (v). None of these factors individually compromises the viability of healthy neurons, but their combination increases the susceptibility to any additional exogenous stressor. This is also the case with the highly abundant protein $\alpha$-synuclein (ASYN), which is expressed widely throughout the nervous system. Whereas the native protein most likely performs antioxidant and protective functions, it engages in multiple reciprocal interactions with cytosolic DA, elevated calcium, or surrounding glia, and the unique combination of ASYN, Cav1.3, and DA might represent a major susceptibility factor for nigrostriatal neurons.

calbindin than do neurons in the MPTP-sensitive substantia nigra pars compacta (SNpc), which agrees with the possibility that calbindin confers resistance against degeneration in the MPTP model [63]. Notably, the ranking of DA neuron susceptibility in the retrorubral area (A8), substantia nigra (A9), and VTA (A10) inversely correlates with calbindin expression in the respective regions in both mice and monkeys [64]. Moreover, the same relative ranking is found in PD and in the human brain, and correlates with the pattern of calbindin expression $[63,65]$.

A major development in research on the relationship between $\mathrm{Ca}^{2+}$ homeostasis and selective neurodegeneration was the identification of an autonomous pacemaking activity of SNpc DA neurons and its reliance on the L-type $\mathrm{Ca}^{2+}$ channel $\mathrm{Ca}_{v} 1 \cdot 3[66,67]$. PD is associated with cell loss in not only the SNpc, but also the locus coeruleus and hypothalamic tuberomammillary nucleus [68]. Intriguingly, these other two regions also exhibit autonomous pacemaking activity, again mediated by $\mathrm{Ca}_{v} 1.3[69,70]$. These observations reveal a potential correlation between the use of extracellular $\mathrm{Ca}^{2+}$ for pacemaking and elevated sensitivity both in PD and in MPTP models. For instance, olfactory bulb dopaminergic neurons are relatively spared from degeneration in PD and in the MPTP model; unlike SNpc cells, these neurons express T-type $\mathrm{Ca}^{2+}$ channels and do not rely on oscillatory $\mathrm{Ca}^{2+}$ waves for pacemaking activity [71]. Analysis of the pacemaking activity of VTA neurons, a population that displays higher resilience than does its nigrostriatal counterpart, indicated their reliance on $\mathrm{Na}^{+}$instead of $\mathrm{Ca}^{2+}$ channels [72]. 
Involvement of extracellular $\mathrm{Ca}^{2+}$ for pacemaking is demanding metabolically, because export of the divalent ion $\mathrm{Ca}^{2+}$ requires a higher ATP investment per charge than does the use of monovalent ions $[61,73]$. A correlation between pacemaking-dependent energy expenditure and MPTP vulnerability was elegantly demonstrated in a cell model in which endogenous $\mathrm{Ca}_{\mathrm{v}} 1.3$ was either pharmacologically inhibited or genetically ablated; both conditions resulted in a 'rejuvenation' of neurons, which indicated that their pacemaking activity switched from a dependence on $\mathrm{Ca}_{v} 1.3$ to a dependence on voltage-gated $\mathrm{Na}^{+}$channels [74]. These rejuvenated neurons were considerably more resistant to MPTP/MPP ${ }^{+}$or rotenone toxicity than were their $\mathrm{Ca}_{v} 1.3$-expressing counterparts $[74,75]$. Collectively, these observations identified $\mathrm{Ca}_{v} 1.3$ channels as potential targets for therapeutic intervention in PD. Notably, human data on how chronic treatment with $\mathrm{Ca}^{2+}$-channel blockers might affect PD development are already available, because these compounds are frequently administered to treat patients with hypertension. Analysis of these initial clinical and epidemiological data indicated that PD risk was reduced in patients chronically treated with $\mathrm{Ca}^{2+}$-channel blockers $[76,77]$.

\section{Morphology of SNpc DA Neurons As a Determinant of $\mathrm{MPP}^{+}$Toxicity}

The pacemaker-associated oscillatory $\mathrm{Ca}^{2+}$ influx is not the only intrinsic feature underlying increased energy demand: the unique architecture of SNpc DA neurons, which had long been largely ignored, is currently suggested to represent another essential feature that contributes to selective neurodegeneration in PD and in the MPTP model [78] (Figure 5). Nigrostriatal DA neurons are characterized by unmyelinated, highly branched axons that can have a total length of up to $0.5 \mathrm{~m}(500000 \mu \mathrm{m})$ per human neuron [79,80]. To maintain such long projections, neurons must expend a high amount of energy, which predisposes the neurons to an energy crisis in the presence of metabolic inhibitors. Moreover, the number of synapses formed by nigrostriatal DA neurons is at least two orders of magnitude higher than that formed by the less vulnerable DA neurons in the VTA [78,80,81]. The rat SNpc contains approximately 12000 DA neurons [80], each of which forms approximately 100 000-250 000 synapses; in the human brain, this is another tenfold higher (1 million-2.4 million synapses per neuron) [78,82]. A computational model of nigrostriatal DA neurons indicated that the neurite-tree size in combination with the high synapse number represents a notable burden for the cellular energy budget: the energy cost increases exponentially with the total length and the degree of branching of the synaptic field [83]. Consequently, nigrostriatal DA neurons continuously face an energy demand that is barely met by the oxidative capacity of their mitochondria [84]. These anatomical features, in addition to the high energy requirement created by $\mathrm{Ca}_{v} 1.3$-mediated influx of extracellular $\mathrm{Ca}^{2+}$, render SNpc DA neurons energetically 'on the edge' [78]. In such a scenario, even moderate stressors could lead to an imbalance of cellular energy provision and consumption (Figures 3 and 5). The concept of a low residual energy capacity could also provide a mechanistic basis for explaining the selective loss of nigrostriatal DA neurons in models of systemically applied rotenone, which, unlike $\mathrm{MPP}^{+}$, is not selectively accumulated in defined cell types.

\section{Synergy of Alpha-Synuclein and Other Susceptibility Factors As a Driving Force}

Besides the unique calcium handling and morphology of nigrostriatal neurons, other susceptibility factors have been proposed. One of the important contributors is $\alpha$-synuclein (ASYN). ASYN has become recognized over the past two decades as a key player in PD pathogenetic processes [85-87]. The protein is expressed at high levels in normal brains, is a primary constituent of Lewy bodies (the intraneuronal inclusions found in most PD brains), and can spread in a prion-like pathology across cells $[85,88]$. Single-point mutations, as well as multiplications of the gene encoding ASYN (SNCA), are causally linked to familial PD. Besides the genetic associations of PD and ASYN, there is also an intriguing relationship between ASYN and the outcome of PD-relevant toxic exposures, such as to MPTP. Vila and colleagues were 
the first to note that administration of MPTP to mice induced an upregulation of ASYN within nigrostriatal dopaminergic neurons [89], a finding confirmed by subsequent studies in nonhuman primate brains [90]. It is noteworthy that enhancement of intraneuronal ASYN concentrations, even when transitory, could trigger pathological processes such as the formation of aggregated ASYN and interneuronal ASYN transmission [91,92]. Thus, findings in the MPTP model are consistent with the possibility that toxic exposures contribute to PD pathogenesis by modifying ASYN proteostasis. Accordingly, reduced ASYN expression led to resistance to MPTP and rotenone [93,94], whereas overexpression resulted in sensitization [95]. Notably, ASYN level alone cannot explain the relative sensitivities of various catecholaminergic neurons; several brain areas that are highly resistant to MPTP express ASYN at similar levels as nigrostriatal neurons, and DA neurons of the (MPP ${ }^{+}$-resistant) VTA express higher levels of ASYN than do (MPP ${ }^{+}$-sensitive) noradrenergic neurons of the locus coeruleus [96]. Other key variables besides expression also affect ASYN neurotoxicity, including turnover, aggregation state, intraneuronal localization, and post-translational modifications. ASYN phosphorylation and nitration are typically seen in postmortem PD brains $[88,97]$ or in animals treated with MPTP [98], while modified ASYN is not detectable in the nigrostriatal system of untreated animals or healthy humans. Modified and/or augmented ASYN is likely to affect neuronal susceptibility to MPTP, and it might do so in concert with other factors, such as neuronal DA content and $\mathrm{Ca}^{2+}$-channel expression $[99,100]$. ASYN enhances cytosolic DA levels by interfering with DA sequestration [101], and interaction of ASYN with DA potently increases neurotoxicity [102]. These observations not only underscore the relationship between MPTP and ASYN, but also illustrate the concept that specific susceptibility factors might separately be insufficient to cause selective neuronal damage, while a combination of these factors could produce additive or synergistic effects that ultimately lead to the demise of defined neuronal subpopulations [99].

\section{Concluding Remarks}

When precisely nigrostriatal DA neurons start dying in PD is unknown, but PD progression, which takes years, is likely driven by a vicious cycle of events, including mitochondrial function disturbances, cellular proteostasis breakdown, increased oxidative stress, and neuroinflammation [88]. Conversely, a defined molecular initiating event (complex I inhibition) (Figure 3) is recognized in MPTP-induced neurodegeneration, characterized by cell death that is completed within a few days. Despite these differences, this MPTP model involves toxic vicious cycles that might recapitulate neurodegenerative mechanisms relevant to the human disease. To understand the extent to which these similarities and differences between the MPTP model and PD are relevant for pharmacological predictions, it is critical to not only obtain a complete overview of the pathomechanisms, but also integrate the latest observations on MPTP bioactivation and distribution. This review was designed to update the reader on information that substantially departs from current textbook knowledge regarding astrocytic $\mathrm{MPP}^{+}$generation and DA neuron degeneration following $\mathrm{MPP}^{+}$uptake. The importance of such considerations for therapeutic research is indicated by the frequent use of MPTP models in not only mechanistic investigations, but also pharmacological intervention studies. The MPTP model has been instrumental in the development of synthetic DA receptor agonists, deep-brain stimulation, DA augmentation approaches, and several novel intervention strategies currently in clinical trials $[19,22]$. The design of a new generation of therapeutic strategies depends on detailed insights into the key events that drive the demise of DA neurons, and on factors, such as the MPTP administration protocol applied, that control such events [103,104]. Systemic integration of available literature data into a framework of molecular key events and their respective relationships is currently being pursued according to the concept of adverse outcome pathways [105,106] (Figure 6). The growing body of identified endogenous susceptibility and resilience factors, together with enhanced knowledge regarding how PD-associated molecular events are reflected by the MPTP model, will allow the development of highly predictive human

\section{Outstanding Questions}

In MPTP models, what is the relative contribution of distinct cell death mechanisms (mitochondrial energyproduction impairment, ROS formation, etc.)? Which mechanism might most efficiently be counteracted to achieve neuroprotection?

Are cell death mechanisms and pathological features similar in acute versus chronic MPTP exposure paradigms? How are single neurons affected by acute and/or chronic MPTP exposure?

Neurodegeneration is complete in a few days or weeks in MPTP models, but nigrostriatal degeneration progresses over years in PD. Does degeneration speed differ at the single cell level? Would chronic MPTP-exposure paradigms closely mimic PD pathogenetic changes?

Nigrostriatal degeneration is the main, but not the only pathological feature of MPTP models. Thus, extranigral MPTP pathology must be examined to answer key questions such as: how much does degeneration of other neuronal populations contribute to MPTPinduced behavioral phenotypes?

To what extent are behavioral parameters assessed in mice (motor activity, pole test, etc.) representative of parkinsonian motor deficits (e.g., bradykinesia)?

What relative roles do various neuroinflammation mechanisms have in acute and/or subacute MPTP treatmentinduced nigrostriatal degeneration?

Aging might affect MPTP toxicity in mice by upregulating MAO-B and, thus, $\mathrm{MPP}^{+}$production. Do the same aging-related changes increase nigrostriatal susceptibility to PD pathogenesis and affect outcome in MPTP models?

How does the growing panel of PDassociated proteins (ASYN, PINK1, LRRK2, etc.) contribute to the events leading to selective tissue injury and neurodegeneration in MPTP models? Are the roles of the proteins in MPTP models relevant to our understanding of their roles in idiopathic PD?

Which specific perturbations of proteostasis link mitochondrial inhibition 


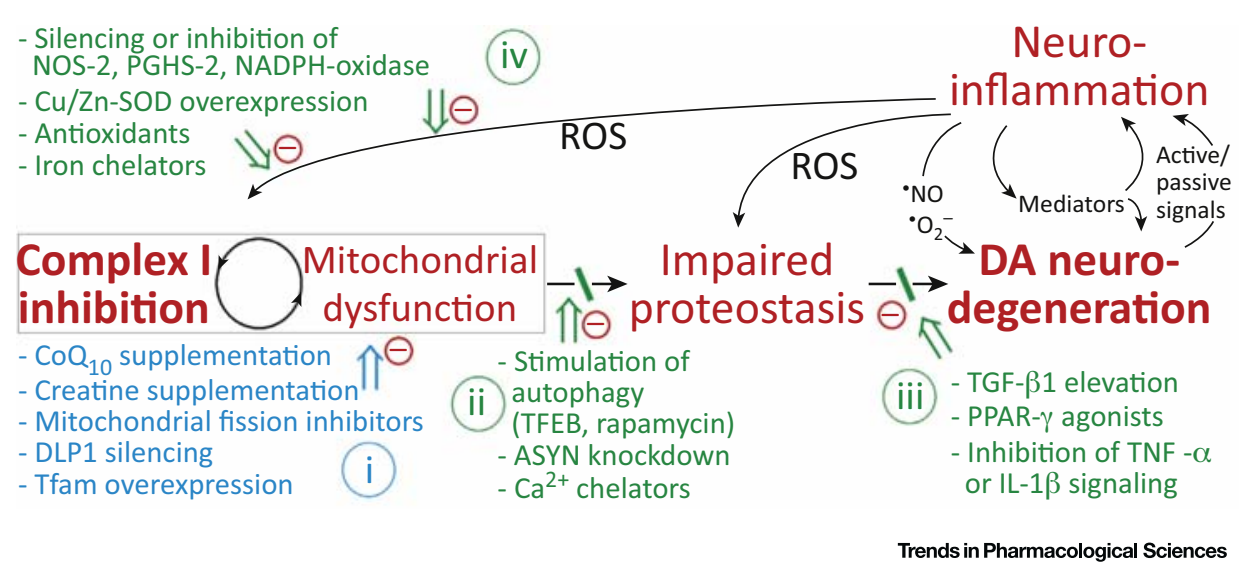

Figure 6. Protection against 1-Methyl-4-Phenyl-1,2,3,6-Tetrahydropyridine (MPTP) Toxicity. The concept of adverse outcome pathways (AOPs) has been introduced to organize the vast array of information on toxicity pathways. MPTP is the archetypical example toxicant in a recently assembled AOP linking the inhibition of mitochondrial complex I with parkinsonian motor deficits. A modified version (including feedback loops) is depicted herein to illustrate known intervention strategies. (i) Several strategies interfere with the first steps of the pathogenic cascade. Given that the MPTP model is used to identify pharmacological treatments that could halt neurodegeneration in PD, intervention strategies that target downstream events of the AOP are of particular interest. Such strategies might prevent the adverse outcome despite not targeting either disease initiation or initial damage. (ii) One group of interventions blocks disturbances of cellular proteostasis downstream of mitochondrial dysfunction. (iii) A second group targets the self-amplifying cycle of neuronal damage and neuroinflammation. This might be achieved by the blocking of proinflammatory mediators or their signaling (e. g., IL-1 $\beta$ ), or by the augmentation of anti-inflammatory conditions (e.g., by elevation of TGF- $\beta 1$ or through PPAR- $\gamma$ agonists). (iv) A final target is reactive oxygen species (ROS), derived from, for example, NADPH oxidase, as well as reactive nitrogen species [e.g., from inducible nitric oxide synthase (NOS-2)]. Alternatively, the cellular antioxidative capacity (Cu/ Zn-SOD overexpression) might be augmented. Notably, oxidative stress might be involved both in the transition of mitochondrial dysfunction to impaired proteostasis, and in several enhancing feedback loops (e.g., complex I inhibition through reversible S-nitrosation or irreversible nitration). Abbreviations: $A S Y N$, alpha synuclein; $C_{10} Q_{10}$, coenzyme $Q_{10} ; C u /$ Zn-SOD, Cu/Zn-superoxide dismutase; DLP-1, dynamin-like protein-1; NOS-2, nitric oxide synthase-2; PGHS-2, prostaglandin endoperoxide $\mathrm{H} 2$ synthase-2; PPAR, peroxisome proliferator activated receptor; Tfam, transcription factor $\mathrm{A}$ mitochondrial; TFEB, transcription factor EB; TGF- $\beta 1$, transforming growth factor $\beta 1$.

cell-based in vitro PD models, and will also provide the framework for developing entirely novel types of PD intervention.

\section{Acknowledgments}

This work was supported by RTG 1331, BMBF, the Doerenkamp-Zbinden Foundation, KoRS Chemical Biology, and the Collaborative Research Center 969 'Chemical and Biological Principles of Cellular Proteostasis', funded by the Deutsche Forschungsgemeinschaft.

\section{References}

1. Langston, J.W. et al. (1983) Chronic Parkinsonism in humans due to a product of meperidine-analog synthesis. Science 219 979-980

2. Di Monte, D.A. et al. (1991) Biotransformation of 1-methyl-4phenyl-1,2,3,6-tetrahydropyridine in primary cultures of mouse astrocytes. J. Pharmacol. Exp. Ther. 258, 594-600

3. Di Monte, D.A. et al. (1992) Production and disposition of 1 methyl-4-phenylpyridinium in primary cultures of mouse astrocytes, Glia 5, 48-55

4. Heikkila, R.E. et al. (1984) Protection against the dopaminergic neurotoxicity of 1 -methyl-4-phenyl-1,2,5,6-tetrahydropyridine by monoamine oxidase inhibitors. Nature 311, 467-469

5. Javitch, J.A. et al. (1985) Parkinsonism-inducing neurotoxin, Nmethyl-4-phenyl-1,2,3,6-tetrahydropyridine: uptake of the metabolite $\mathrm{N}$-methyl-4-phenylpyridine by dopamine neurons explains selective toxicity. Proc. Natl. Acad. Sci. U. S. A. 82 , 2173-2177
6. Ramsay, R.R. and Singer, T.P. (1986) Energy-dependent uptake of $\mathrm{N}$-methyl-4-phenylpyridinium, the neurotoxic metabolite of 1-methyl-4-phenyl-1,2,3,6-tetrahydropyridine, by mitochondria. J. Biol. Chem. 261, 7585-7587

7. Schildknecht, S. et al. (2009) Requirement of a dopaminergic neuronal phenotype for toxicity of low concentrations of 1 methyl-4-phenylpyridinium to human cells. Toxicol. Appl. Pharmacol. 241, 23-35

8. Scholz, D. et al. (2011) Rapid, complete and large-scale generation of post-mitotic neurons from the human LUHMES cell line. J Neurochem. 119, 957-971

9. Aguirre, P et al. (2012) The dopamine metabolite aminochrome inhibits mitochondrial complex I and modifies the expression of iron transporters DMT1 and FPN1. Biometals 25, 795-803

10. Pöltt, D. et al. (2012) Uncoupling of ATP-depletion and cell death in human dopaminergic neurons. Neurotoxicology 33 769-779 to the downstream consequence of MPTP exposure (MPP ${ }^{+}$neurotoxicity), and what functions do ASYN and other PD-related proteins perform in this key event? 
11. Sherer, T.B. et al. (2007) Mechanism of toxicity of pesticides acting at complex $\mid$ : relevance to environmental etiologies of Parkinson's disease. J. Neurochem. 100, 1469-1479

12. Sherer, T.B. et al. (2003) Mechanism of toxicity in rotenone models of Parkinson's disease. J. Neurosci. 23, 10756-10764

13. Przedborski, S. et al. (1992) Transgenic mice with increased Cu/ $\mathrm{Zn}$-superoxide dismutase activity are resistant to $\mathrm{N}$-methyl-4phenyl-1,2,3,6-tetrahydropyridine-induced neurotoxicity. J. Neurosci. 12, 1658-1667

14. Zhang, J. et al. (2000) Enhanced N-methyl-4-phenyl-1,2,3,6tetrahydropyridine toxicity in mice deficient in CuZn-superoxide dismutase or glutathione peroxidase. J. Neuropathol. Exp. Neurol. 59, 53-61

15. Tanaka, S. et al. (2013) Activation of microglia induces symptoms of Parkinson's disease in wild-type, but not in IL-1 knockout mice. J. Neuroinflammation 10, 143

16. Mount, M.P. et al. (2007) Involvement of interferon-gamma in microglial-mediated loss of dopaminergic neurons. J. Neurosci. 27, 3328-3337

17. Sriram, K. et al. (2002) Mice deficient in TNF receptors are protected against dopaminergic neurotoxicity: implications for Parkinson's disease. FASEB J. 16, 1474-1476

18. Qi, X. et al. (2013) A novel Drp1 inhibitor diminishes aberrant mitochondrial fission and neurotoxicity. J. Cell Sci. 126, 789-802

19. Rappold, P.M. et al. (2014) Drp1 inhibition attenuates neurotoxicity and dopamine release deficits in vivo. Nat. Commun. 5, 5244

20. Santos, D. et al. (2015) The impact of mitochondrial fusion and fission modulation in sporadic Parkinson's disease. Mol. Neurobiol. 52, 573-586

21. Wang, X. et al. (2011) DLP1-dependent mitochondrial fragmentation mediates 1-methyl-4-phenylpyridinium toxicity in neurons: implications for Parkinson's disease. Aging Cell 10, 807-823

22. Uchida, S, et al. (2015) The adenosine A2A receptor antagonist, istradefylline enhances the anti-parkinsonian activity of low doses of dopamine agonists in MPTP-treated common marmosets. Eur. J. Pharmacol. 747, 160-165

23. Hikishima, K. et al. (2015) Voxel-based morphometry of the marmoset brain: In vivo detection of volume loss in the substantia nigra of the MPTP-treated Parkinson's disease model. Neuroscience 300, 585-592

24. German, D.C. etal. (1996) The neurotoxin MPTP causes degeneration of specific nucleus A8, A9 and A10 dopaminergic neurons in the mouse. Neurodegeneration 5, 299-312

25. German, D.C. et al. (1992) Disease-specific patterns of locus coeruleus cell loss. Ann. Neurol. 32, 667-676

26. Shen, R.S. et al. (1985) Serotonergic conversion of MPTP and dopaminergic accumulation of MPP+ . FEBS Lett. 189, 225-230

27. Russell, S.M. et al. (1979) The vectorial orientation of human monoamine oxidase in the mitochondrial outer membrane. Biochem. J. 181, 7-14

28. Vincent, S.R. (1989) Histochemical localization of 1-methyl-4phenyl-1,2,3,6-tetrahydropyridine oxidation in the mouse brain. Neuroscience 28, 189-199

29. Jackson-Lewis, V. et al. (1995) Time course and morphology of dopaminergic neuronal death caused by the neurotoxin 1 methyl-4-phenyl-1,2,3,6-tetrahydropyridine. Neurodegeneration 4, 257-269

30. Efremova, L. et al. (2015) Prevention of the degeneration of human dopaminergic neurons in an astrocyte co-culture system allowing endogenous drug metabolism. Br. J. Pharmacol. 172, 4119-4132

31. Kitayama, S. et al. (1998) MPP+ toxicity and plasma membrane dopamine transporter: study using cell lines expressing the wildtype and mutant rat dopamine transporters. Biochim. Biophys. Acta 1404, 305-313

32. Schildknecht, S. et al. (2015) Preferential extracellular generation of the active parkinsonian toxin MPP+ by transporter-independent export of the intermediate MPDP+ . Antioxid. Redox Signal. 23, 1001-1016
33. Cui, M. et al. (2009) The organic cation transporter-3 is a pivotal modulator of neurodegeneration in the nigrostriatal dopaminergic pathway. Proc. Natl. Acad. Sci. U. S. A. 106, 8043-8048

34. Rappold, P.M. et al. (2011) Paraquat neurotoxicity is mediated by the dopamine transporter and organic cation transporter-3. Proc. Natl. Acad. Sci. U. S. A. 108, 20766-27137

35. Chiba, K. et al. (1984) Metabolism of the neurotoxic tertian amine, MPTP, by brain monoamine oxidase. Biochem. Biophys. Res. Commun. 120, 574-578

36. Korytowski, W. et al. (1987) Mechanism of oxidation of 1 methyl-4-phenyl-2,3-dihydropyridinium (MPDP+). Biochem. Biophys. Res. Commun. 144, 692-698

37. Peterson, L.A. et al. (1985) Studies on the 1-methyl-4-phenyl2,3-dihydropyridinium species 2,3-MPDP+, the monoamine oxidase catalyzed oxidation product of the nigrostriatal toxin 1-methyl-4-phenyl-1,2,3,6-tetrahydropyridine (MPTP). J. Med. Chem. 28, 1432-1436

38. Mik, E.G. et al. (2006) Mitochondrial PO2 measured by delayed fluorescence of endogenous protoporphyrin IX. Nat. Methods 3 , 939-945

39. Lin, C.J. et al. (2010) Cellular localization of the organic cation transporters, OCT1 and OCT2, in brain microvessel endothelial cells and its implication for MPTP transport across the bloodbrain barrier and MPTP-induced dopaminergic toxicity in rodents. J. Neurochem. 114, 717-727

40. Kadar, H. et al. (2014) MALDI mass spectrometry imaging of 1-methyl-4-phenylpyridinium $\left(\mathrm{MPP}^{+}\right.$) in mouse brain. Neurotox Res. 25, 135-145

41. Grimsby, J. et al. (1997) Increased stress response and beta-phenylethylamine in MAOB-deficient mice. Nat. Genet. 17, 206-210

42. Uehara, S. et al. (2015) Activation and deactivation of 1-methyl4-phenyl-1,2,3,6-tetrahydropyridine by cytochrome P450 enzymes and flavin-containing monooxygenases in common marmosets (Callithrix jacchus). Drug Metab. Dispos. 43, 735-742

43. Bajpai, P. et al. (2013) Metabolism of 1-methyl-4-phenyl1,2,3,6-tetrahydropyridine by mitochondrion-targeted cytochrome P450 2D6: implications in Parkinson disease. J. Biol. Chem. 288, 4436-4451

44. Lu, Y et al. (2013) CYP2D6*4 allele polymorphism increases the risk of Parkinson's disease: evidence from meta-analysis. PLOS One 8, e84413

45. Elbaz, A. et al. (2004) CYP2D6 polymorphism, pesticide exposure, and Parkinson's disease. Ann. Neurol. 55, 430-434

46. Ingelman-Sundberg, M. (2005) Genetic polymorphisms of cytochrome P450 2D6 (CYP2D6): clinical consequences, evolutionary aspects and functional diversity. Pharmacogenomics J. 5, $6-13$

47. Herraiz, T. et al. (2013) Metabolite profile resulting from the activation/inactivation of 1-methyl-4-phenyl-1,2,3,6-tetrahydropyridine and 2 -methyltetrahydro- $\beta$-carboline by oxidative enzymes. Biomed. Res. Int. 2013, 248608

48. Herraiz, T. et al. (2006) Comparative aromatic hydroxylation and $\mathrm{N}$-demethylation of MPTP neurotoxin and its analogs, $\mathrm{N}$-methylated beta-carboline and isoquinoline alkaloids, by human cytochrome P450 2D6. Toxicol. Appl. Pharmacol. 216, 387-398

49. Corsini, G.U. et al. (1985) 1-Methyl-4-phenyl-1,2,3,6-tetrahydropyridine (MPTP) neurotoxicity in mice is enhanced by pretreatment with diethyldithiocarbamate. Eur. J. Pharmacol. 119, 127-128

50. Mann, A. and Tyndale, R.F. (2010) Cytochrome P450 2D6 enzyme neuroprotects against 1-methyl-4-phenylpyridinium toxicity in SH-SY5Y neuronal cells. Eur. J. Neurosci. 31, 1185-1193

51. Chiba, K et al (1990) Effects of N-methylmercaptoimidazole on the disposition of MPTP and its metabolites in mice. Eur. Pharmacol. 180, 59-67

52. Fuller, R.W. et al. (1989) Tissue concentrations of MPTP and $\mathrm{MPP}^{+}$in relation to catecholamine depletion after the oral or subcutaneous administration of MPTP to mice. Life Sci. 45 2077-2083 
53. Masoud, S.T. et al. (2015) Increased expression of the dopamine transporter leads to loss of dopamine neurons, oxidative stress and I-DOPA reversible motor deficits. Neurobiol. Dis. 74, 66-75

54. Lohr, K.M. et al. (2014) Increased vesicular monoamine transporter enhances dopamine release and opposes Parkinson disease-related neurodegeneration in vivo. Proc. Natl. Acad. Sci. U. S. A. 111, 9977-9982

55. Gainetdinov, R.R. et al. (1998) Increased MPTP neurotoxicity in vesicular monoamine transporter 2 heterozygote knockout mice. J. Neurochem. 70, 1973-1978

56. German, C.L. et al. (2015) Regulation of the dopamine and vesicular monoamine transporters: pharmacological targets and implications for disease. Pharmacol. Rev. 67, 10051024

57. Taylor, T.N. et al. (2014) Reduced vesicular storage of catecholamines causes progressive degeneration in the locus ceruleus. Neuropharmacology 76, 97-105

58. Brighina, L. et al. (2013) Analysis of vesicular monoamine transporter 2 polymorphisms in Parkinson's disease. Neurobiol. Aging 34, 1712

59. Yang, X. et al. (2015) Polymorphism in the vesicular monoamine transporter 2 gene decreases the risk of Parkinson's disease in Han Chinese men. Parkinsons Dis. 2015, 903164

60. Richardson, J.R. et al. (2006) Developmental exposure to the pesticide dieldrin alters the dopamine system and increases neurotoxicity in an animal model of Parkinson's disease. FASEB J. 20, 1695-1697

61. Surmeier, D.J. and Schumacker, P.T. (2013) Calcium, bioenergetics, and neuronal vulnerability in Parkinson's disease. J. Biol. Chem. 288, 10736-10741

62. Chen, T. et al. (2013) Homer1 knockdown protects dopamine neurons through regulating calcium homeostasis in an in vitro model of Parkinson's disease. Cell Signal. 25, 28632870

63. Dopeso-Reyes, I.G. (2014) Calbindin content and differential vulnerability of midbrain efferent dopaminergic neurons in macaques. Front. Neuroanat. 8, 146

64. Yuan, H.H. et al. (2013) The neuroprotective effect of overexpression of calbindin-D(28k) in an animal model of Parkinson's disease. Mol. Neurobiol. 47, 117-122

65. Hurley, M.J. et al. (2013) Parkinson's disease is associated with altered expression of CaV1 channels and calcium-binding proteins. Brain 136, 2077-2097

66. Nedergaard, S. et al. (1993) Nifedipine- and omega-conotoxinsensitive $\mathrm{Ca}^{2+}$ conductances in guinea-pig substantia nigra pars compacta neurones. J. Physiol. 466, 727-747

67. Guzman, J.N. et al. (2009) Robust pacemaking in substantia nigra dopaminergic neurons. J. Neurosci. 29, 11011-11019

68. Braak, H. et al. (2004) Stages in the development of Parkinson's disease-related pathology. Cell Tissue Res. 318, 121-134

69. Stevens, D.R. and Haas, H.L. (1996) Calcium-dependent prepotentials contribute to spontaneous activity in rat tuberomammillary neurons. J. Physiol. 493, 747-754

70. Matschke, L.A. et al. (2015) A concerted action of L-and T-type $\mathrm{Ca}^{2+}$ channels regulates locus coeruleus pacemaking. Mol. Cell. Neurosci. 68, 293-302

71. Pignatelli, A. et al. (2005) Functional properties of dopaminergic neurones in the mouse olfactory bulb. J. Physiol. 564, 501-514

72. Khaliq, Z.M. and Bean, B.P. (2010) Pacemaking in dopaminergic ventral tegmental area neurons: depolarizing drive from background and voltage-dependent sodium conductances. J. Neurosci. 30, 7401-7413

73. Surmeier, D.J. et al. (2011) The role of calcium and mitochondrial oxidant stress in the loss of substantia nigra pars compacta dopaminergic neurons in Parkinson's disease. Neuroscience 198, 221-231

74. Chan, C.S. et al. (2007) 'Rejuvenation' protects neurons in mouse models of Parkinson's disease. Nature 447, 1081-1086

75. Hijic, E. et al. (2011) The L-type channel antagonist isradipine is neuroprotective in a mouse model of Parkinson's disease. Neurobiol. Dis. 43, 364-371
76. Gudala, K. et al. (2015) Reduced risk of Parkinson's disease in users of calcium channel blockers: a meta-analysis. Int. J. Chronic Dis. 2015, 697404

77. Lang, Y. et al. (2015) Calcium channel blocker use and risk of Parkinson's disease: a meta-analysis. Pharmacoepidemiol. Drug Saf. 24, 559-566

78. Bolam, J.P. and Pissadaki, E.K. (2012) Living on the edge with too many mouths to feed: why dopamine neurons die. Mov. Disord. 27, 1478-1483

79. Matsuda, W. et al. (2009) Single nigrostriatal dopaminergic neurons form widely spread and highly dense axonal arborizations in the neostriatum. J. Neurosci. 29, 444-453

80. Nair-Roberts, R.G. (2008) Stereological estimates of dopaminergic, GABAergic and glutamatergic neurons in the ventra tegmental area, substantia nigra and retrorubral field in the rat. Neuroscience 152, 1024-1031

81. Brichta, L. and Greengard, P. (2014) Molecular determinants of selective dopaminergic vulnerability in Parkinson's disease: an update. Front. Neuroanat. 8, 152

82. Hardman, C.D. et al. (2002) Comparison of the basal ganglia in rats, marmosets, macaques, baboons, and humans: volume and neuronal number for the output, internal relay, and striatal modulating nuclei. J. Comp. Neurol. 445, 238-255

83. Pissadaki, E.K. and Bolam, J.P. (2013) The energy cost of action potential propagation in dopamine neurons: clues to susceptibility in Parkinson's disease. Front. Comput. Neurosci. 7, 13

84. Harris, J.J. et al. (2012) Synaptic energy use and supply. Neuron $75,762-777$

85. Goedert, M. et al. (2017) Like prions: the propagation of aggregated tau and $\alpha$-synuclein in neurodegeneration. Brain 140, 266-278

86. Goedert, M. et al. (2017) The synucleinopathies: twenty years on. J. Parkinsons Dis. 7, S53-S71

87. Braak, H. and Del Tredici, K. (2017) Neuropathological staging of brain pathology in sporadic Parkinson's disease: separating the wheat from the chaff. J. Parkinsons Dis. 7, S73-S87

88. Schildknecht, S. et al. (2013) Oxidative and nitrative alpha-synuclein modifications and proteostatic stress: implications for disease mechanisms and interventions in synucleinopathies. J. Neurochem. 125, 491-511

89. Vila, M. et al. (2000) Alpha-synuclein up-regulation in substantia nigra dopaminergic neurons following administration of the parkinsonian toxin MPTP. J. Neurochem. 74, 721-729

90. Purisai, M.G. et al. (2005) Alpha-synuclein expression in the substantia nigra of MPTP-lesioned non-human primates. Neurobiol. Dis. 20, 898-906

91. Ulusoy, A. et al. (2013) Caudo-rostral brain spreading of $\alpha$-synuclein through vagal connections. EMBO Mol. Med. 5, 11191127

92. Helwig, M. et al. (2016) Brain propagation of transduced $\alpha$-synuclein involves non-fibrillar protein species and is enhanced in $\alpha$-synuclein null mice. Brain 139, 856-870

93. Zharikov, A.D. et al. (2015) shRNA targeting $\alpha$-synuclein prevents neurodegeneration in a Parkinson's disease model. J. Clin. Invest. 125, 2721-2735

94. Dauer, W. et al. (2015) Resistance of alpha-synuclein null mice to the parkinsonian neurotoxin MPTP. Proc. Natl. Acad. Sci. U. S. A. 99, 14524-14529

95. Song, L.K. et al. (2015) Targeted overexpression of $\alpha$-synuclein by rAAV2/1 vectors induces progressive nigrostriatal degeneration and increases vulnerability to MPTP in mouse. PLOS One 10, e0131281

96. Taguchi, K. et al. (2016) Brain region-dependent differentia expression of alpha-synuclein. J. Comp. Neurol. 524, 1236-1258

97. Oueslati, A. et al. (2010) Role of post-translational modifications in modulating the structure, function and toxicity of alpha-synuclein: implications for Parkinson's disease pathogenesis and therapies. Prog. Brain Res. 183, 115-145

98. McCormack, A.L. et al. (2008) Pathologic modifications of alpha-synuclein in 1-methyl-4-phenyl-1,2,3,6-tetrahydropyridine (MPTP)-treated squirrel monkeys. J. Neuropathol. Exp. Neurol. 67, 793-802 
99. Mosharov, E.V. et al. (2009) Interplay between cytosolic dopamine, calcium, and alpha-synuclein causes selective death of substantia nigra neurons. Neuron 62, 218-229

100. Jackson-Lewis, V. and Przedborski, S. (2008) The MPTP mouse model of Parkinson's disease: the true, the false, and the unknown. In Parkinson's Disease: Molecular and Therapeutic Insights from Model Systems (Nass, R. and Przedborski, S., eds), pp. 147-158, Elsevier

101. Lotharius, J. et al. (2002) Effect of mutant alpha-synuclein on dopamine homeostasis in a new human mesencephalic cell line. J. Biol. Chem. 277, 38884-38894

102. Martinez-Vicente, M. (2008) Dopamine-modified alpha-synuclein blocks chaperone-mediated autophagy. J. Clin. Invest. $118,777-788$
103. Bové, J. and Perier, C. (2012) Neurotoxin-based models of Parkinson's disease. Neuroscience 211, 51-76

104. Jackson-Lewis, V. and Przedborski, S. (2007) Protocol for the MPTP mouse model of Parkinson's disease. Nat. Protoc. 2 $141-151$

105. Villeneuve, D.L. (2014) Adverse outcome pathway (AOP) development I: strategies and principles. Toxicol. Sci. 142, 312-320

106. Ockleford, C. et al. (2017) Investigation into experimental toxicological properties of plant protection products having a potential link to Parkinson's disease and childhood leukaemia. EFSA J. 15, 4691 